\title{
Illumination profile characterization of a light applicatorsark
}

\section{Munck *, N. Betrouni, S. Mordon}

INSERM U1189, Lille University Hospital, Lille, France

Background: When surgery is part of a multimodal treatment for malignant pleural mesothelioma, an aggressive tumor of the pleura, it is crucial to combine it with a local adjuvant treatment to kill residual tumour cells. Recently, intrapleural photodynamic therapy (iPDT), has emerged as a promising treatment, with major impact on survival and minimum toxicity [1]. Complete and homo geneous illumination of the pleural cavity is essential to the success of iPDT, but remains a challenge. Knowing the repartition and prop-agation of light around the light device could be the first step towards optimizing dosimetry. Here we propose a characterization method of the illumination profile of a specific light device.

Methods: The light wand was made of a cylindrical diffuser (Medlight ${ }^{\circledR}$ S.A. Switzerland) located inside an endotracheal tube and connected to a medical diode laser $635 \pm 3 \mathrm{~nm}$ (Ceralas ${ }^{\circledR}$, Bio-litec). The wand was fixed horizontally in a tank filled with dilute $0.01 \%$ intralipid. Light dosimetry was performed around the tip of the wand using two complementary methods: accurate measure-ments of light power (nW) with an isotropic probe (Medlight ${ }^{\circledR}$ ) connected to a wattmeter (Newport ${ }^{\circledR}$ 841-PE) and measurements of pixel intensity with digital photography. An effective attenua-tion coefficient ( eff ) was to be defined. Fluence rate measurements were to be confronted with those of a $1 \mathrm{~cm}^{2}$ flat sensor located at the device's surface.

Results: The experimental measurements obtained with the isotropic probe allowed to estimate an effective attenuation coefficient eff $=0.56 \mathrm{~cm}^{-1}$. Combined with the photography's spatial representation, a theoretical illumination profile was established: it showed an ellipse-shaped illumination with iso-surfaces of flu-ence rate, defining a gradient light dose according to the distance from the diffuser. For instance, $20 \%$ of light power is delivered at $23 \mathrm{~mm}$ from the center of the diffuser. Fluence rate obtained with the flat sensor at $14 \mathrm{~mm}$ from the diffuser $\left(0.023 \mathrm{~W} / \mathrm{cm}^{2}\right)$ matched the one calculated from the illumination profile $(0.02$ $\mathrm{W} / \mathrm{cm}^{2}$ ). This work has also been transposed to the same light device, but with a bare optical fiber (FiberTech ${ }^{\circledR}$ ), also used for iPDT.

Conclusion: A theoretical illumination profile of a light wand for iPDT was established and could be used in the future to improve peroperative dosimetry, as part of a spatial tracking system.

\section{Reference}

[1] J.S. Friedberg, Ann. Thorac. Surg. 91 (6) (2011) 1738-1745. 